\title{
Uncertainty Co-Movement in Major European Countries
}

\author{
Aviral Kumar Tiwari', Niyati Bhanja², Arif Billah Dar ${ }^{3}$ \\ ${ }^{1}$ Faculty of Management, IBS Hyderabad, A Constituent of IFHE (Deemed to Be) University, Hyderabad, India \\ ${ }^{2}$ Department of Economics and International Business, University of Petroleum and Energy Studies, \\ Uttarakhand, India \\ ${ }^{3}$ Department of Economic Environment and Strategy, Institute of Management and Technology, Uttar Pradesh, \\ India \\ Email: aviral.eco@gmail.com, niyati.eco@gmail.com, billaharif0@gmail.com
}

Received 12 March 2015; accepted 10 April 2015; published 15 April 2015

Copyright (C) 2015 by authors and Scientific Research Publishing Inc.

This work is licensed under the Creative Commons Attribution International License (CC BY).

http://creativecommons.org/licenses/by/4.0/

(c) (i) Open Access

\begin{abstract}
The recent bouts of crises in the Europe and the US have spurred increasing interest on the comovement of output and stock market across the European countries. The evidence of such comovements is rooted mainly from the spillover of uncertainty among the European countries. Hence, using a new uncertainty index from Baker et al. [1], we investigate both the time-varying as well as frequency based co-movement of uncertainty in the selected European countries. Our results suggest both time and frequency varying co-movement of the uncertainty indices. In particular, these co-movements are found to be more pronounced during the crises periods.
\end{abstract}

\section{Keywords}

Uncertainty Spillover, Cohesion, European Countries, Wavelet

\section{Introduction}

The vast body of literature in economics and finance focuses the co-movement of output, commodity prices and stock prices [2]-[4]. Nevertheless, the knowledge of uncertainty co-movement across nations is much more important than the co-movement of stock prices and output. Since uncertainty incurs costs and loss for the economy, it is useful for policy makers to know the synchronization of uncertainty across countries. The recent emergence of financial crisis has lead to the renewed interest in analyzing the co-movement of output, commodity prices and stock prices. A significant amount of literature focuses on the time and frequency varying co-movement in stock prices and output using wavelets [2] [5] [6]. However, noteworthy point is that the co-movement 
in these macroeconomic variables and cause of economic crisis is the rooted spillover of uncertainty among the more connected countries and this question we address in the present study. ${ }^{1}$ Since our study period is characterized by the periods of crisis and tranquility, the time varying co-movement is of particular interest, because periods of uncertainty and tranquility could indicate different nature of co-movements. The motivation for studying the uncertainty co-movement at different frequencies for this study arises out of the fact that the key drivers of uncertainty like fiscal, monetary and real factors operate at different frequencies. ${ }^{2}$ For example, output uncertainty may transmit to other countries at business, Juglar or Kuznets cycles. Similarly, inflation uncertainty could flow to other countries because shocks to the international prices of the imported goods are not adjusted by exchange rates. These shocks flow to domestic consumer prices over the short to medium frequencies. Yet, the stock market uncertainty may be transmitted at different trading frequencies to other countries by the heterogeneous behavior of investors. Since output, inflation and stock market uncertainty form important components of overall uncertainty, it is important to know how uncertainty is synchronized between countries both over time as well as over frequencies. Thus uncertainty co-movement in our case may be defined in terms of co-movement of uncertainty pertaining to the socio-economic variables. We investigate both of these issues using the wavelet based measure of cohesion for uncertainty indices of selected European countries.

The innovation of this paper has been done in two ways. First, we test the time varying co-movement of uncertainty in the major pair wise European countries. Second, we investigate this co-movement at different frequencies with defined time dynamics. Last but not least major contribution of this study lies in analyzing, for the first time, the co-movement of uncertainty in the major European countries using new index of uncertainty. Our results are interesting and unravel both time varying as well as frequency varying co-movement of uncertainty which could not have been obtained by following the time domain or frequency domain approach only.

The organization of the paper follows as. Section 2, the methodology describing the wavelet based measure of co-movement is described. In Section 3, results are described and discussed. Section 4 draws the conclusion.

\section{Methodology}

The technique of wavelet transformation enables us to understand the evolution of a signal or time series across time as well as over frequency. It adjusts the time resolution to the frequency; narrows down the window width on high frequencies and widens while dealing with low frequencies. It makes use of local base functions that can be translated and stretched into both time and frequency. Moreover, the Wavelets are characterized by finite energy such that they grow and die out within a period. Mathematically, the Wavelets are defined as

$$
\psi_{\tau, s}(t)=\frac{1}{\sqrt{s}} \psi\left(\frac{t-\tau}{s}\right)
$$

where, $\tau$ is the translation parameter, $s$ is the dilation parameter, $\frac{1}{\sqrt{s}}$ is a normalization factor and $\psi_{\tau, s}(t)$ are elementary functions obtained by decomposition of a time series through wavelet transform and are derived from a time-localized mother wavelet $\psi(t)$ [7].

The convolution of continuous wavelet transform (CWT) of a time $x(t)$ series with respect to $\psi(t)$ is given by;

$$
W_{x}(\tau, s)=\int_{-\infty}^{+\infty} x(t) \psi_{\tau, s}^{*}(t) \mathrm{d} t=\frac{1}{\sqrt{s}} \int_{-\infty}^{+\infty} x(t) \psi^{*}\left(\frac{t-\tau}{s}\right) \mathrm{d} t
$$

where, * denotes the complex conjugate.

Moreover, several interesting quantities can be captured within wavelet domain. The measure of wavelet power spectrum that captures the relative contribution at each time and at each scale to the time series' variance is defined as $\left|W_{x}(\tau, s)\right|^{2}$. Further, the measure of cross-wavelet spectrum that captures the covariance between two time series [say, $x(t)$ and $y(t)]$ in the time-frequency space $\left[W_{x}(\tau, s)\right.$ and $\left.W_{y}(\tau, s)\right]$ is given as,

\footnotetext{
${ }^{1}$ Here more connected countries we can look from the point of countries are member of some trade area or have formed monetary union or any such trade blocks or there is high degree of trade dependence without such trade blocks among others.

${ }^{2}$ The fluctuations that last between 2 - 8 years are termed as Business cycles by Rua (2013). Schumpeter (1939) defines 8 - 10 years periodicity cycles as Juglar cycles. Cycles with the periodicity of 18 - 20 years are referred by Samuelson (1998) as Kuznet's Intermediate cycles.
} 
$W_{x y}(\tau, s)=W_{x}(\tau, s) W_{y}^{*}(\tau, s)$.

Following Croux et al. [8] the cross-wavelet spectrum can be decomposed into real and imaginary components defined as,

$$
\rho_{x y}(\tau, s)=\frac{\Re\left(W_{x y}(\tau, s)\right)}{\sqrt{\left|W_{x}(\tau, s)\right|^{2}}\left|W_{y}(\tau, s)\right|^{2}}
$$

where, $\mathfrak{R}$ measures the contemporaneous variance and corresponds to the real part of the cross-wavelet spectrum. The wavelet $\rho_{x y}(\tau, s)$ quantifies the co-movement in the time-frequency space and identifies the timefrequency period over which the co-movement is higher. Basically, the cross-wavelet spectrum acts as a contemporaneous correlation coefficient around each moment in time and for each frequency [2].

The striking feature of cross-wavelet spectrum lies with its ability in providing information about the comovement both at the frequency as well as over time. Moreover, assessing the contour plot of the wavelet cross spectrum it can be identified the time-frequency regions over which the two series commove as well can be assessed the features of the time and frequency variation of the co-movement. The suggested wavelet-based measure, hence, enriches the analysis of co-movement between a set of variables.

\section{Results and Discussion}

We assess the co-movement of uncertainty among the major European countries by using the uncertainty index originally constructed by Baker [1]. We begin by plotting the uncertainty index of all the countries in Figure 1. The plots of uncertainty indices indicate that there is a reasonable amount of co-movement in the uncertainty indices of all the countries. Nevertheless, it is not clear that how the co-movement varies both over time and across frequencies. In order to understand the dynamics of co-movement across both frequencies as well as over time we use the wavelet based measure of cohesion. We estimate the Equation (5) for all the pair of countries in this section. Our results based on the estimation of Equation (5) for all the possible country pairs are shown through contour plots in Figures 2-7. ${ }^{3}$ The vertical axis measures the frequency and horizontal axis measures frequency interpreted in terms of time (years). The colour scale measures cohesion and the ranges of from -1 to +1 . The deep blue colour indicates the perfect negative cohesion and deep red colour indicates the perfect positive cohesion. The identification of time and frequency varying coherence is done by inspecting the contour plot. One can therefore identify both frequency bands (in the vertical axis) and time intervals (in the horizontal axis) where the uncertainty indices are synchronized.

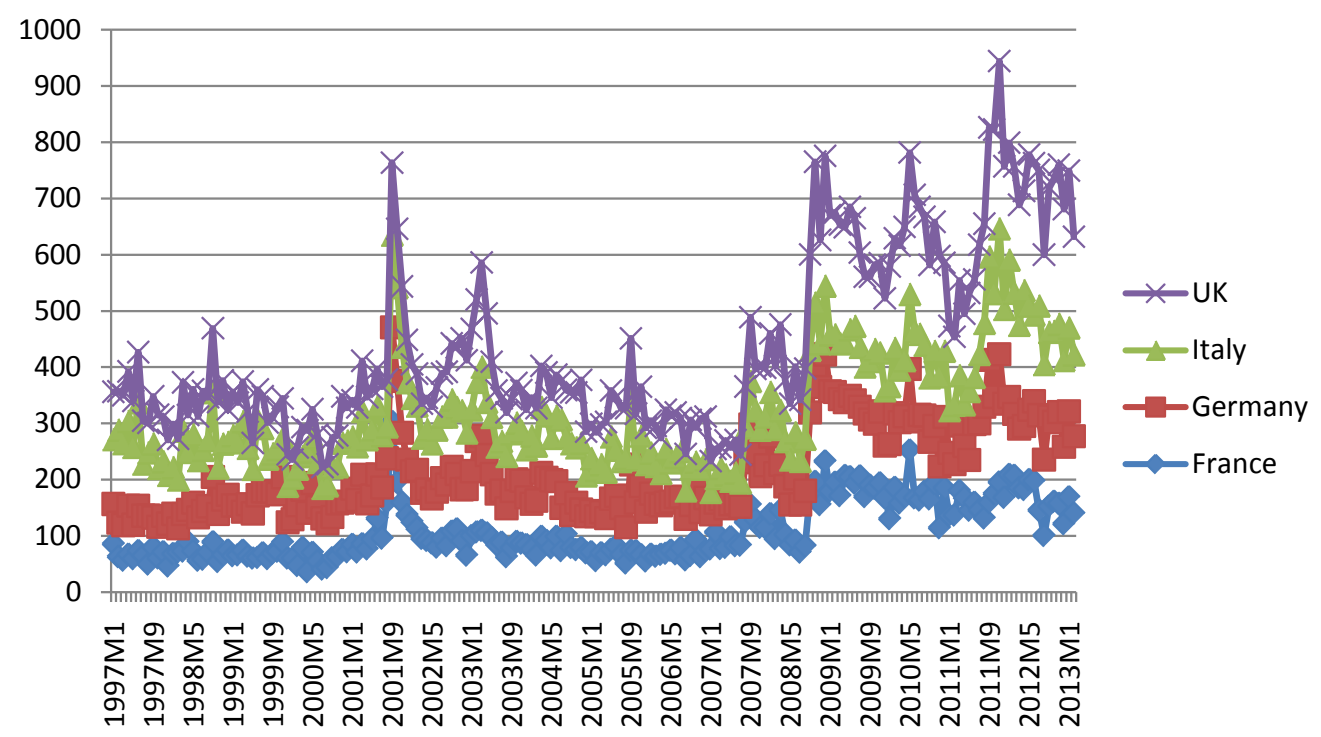

Figure 1. Plot of uncertainty indices.

\footnotetext{
${ }^{3}$ This is because three dimensions (time, frequency and cohesion) cannot be presented on a two dimensional figure.
} 


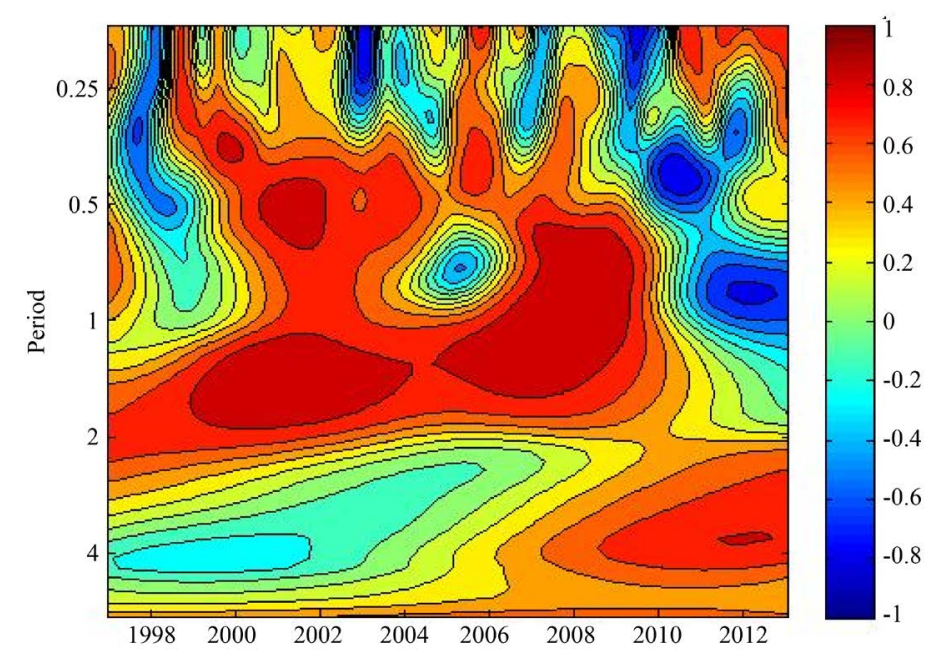

Figure 2. Co-movement between the uncertainty indices of France and Germany.

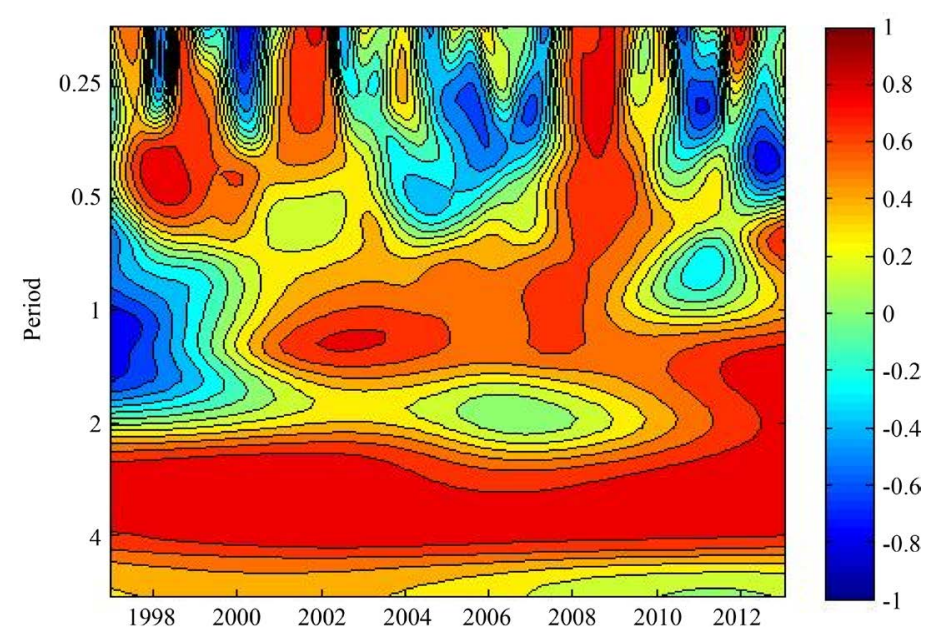

Figure 3. Co-movement between the uncertainty indices of France and Italy.

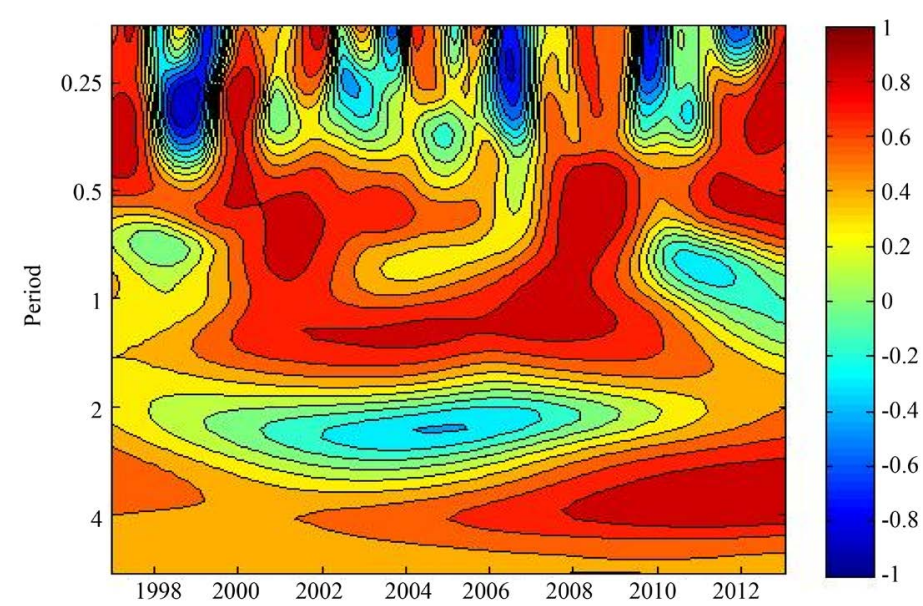

Figure 4. Co-movement between the uncertainty indices of France and UK. 


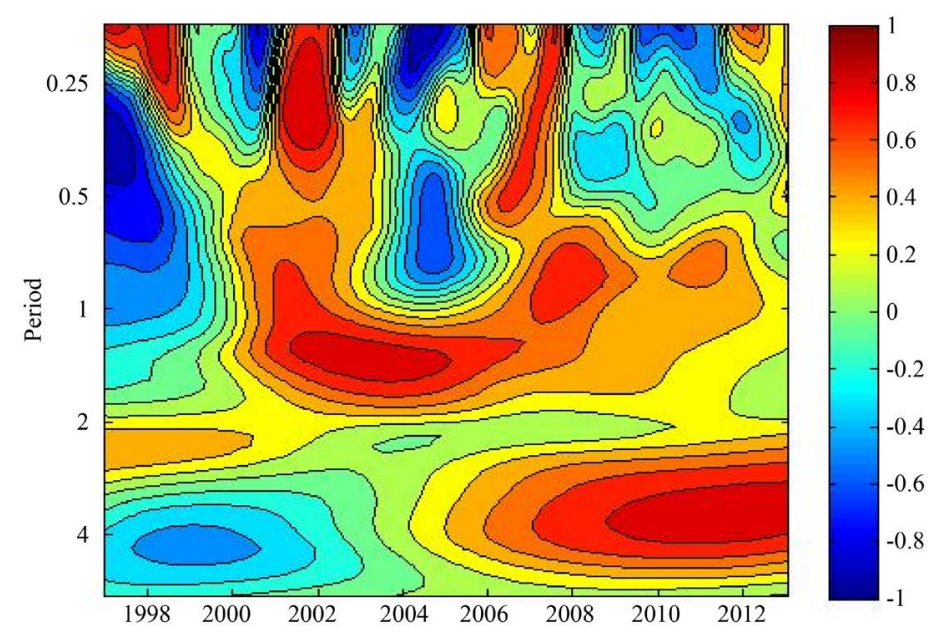

Figure 5. Co-movement between the uncertainty indices of Germany and Italy.

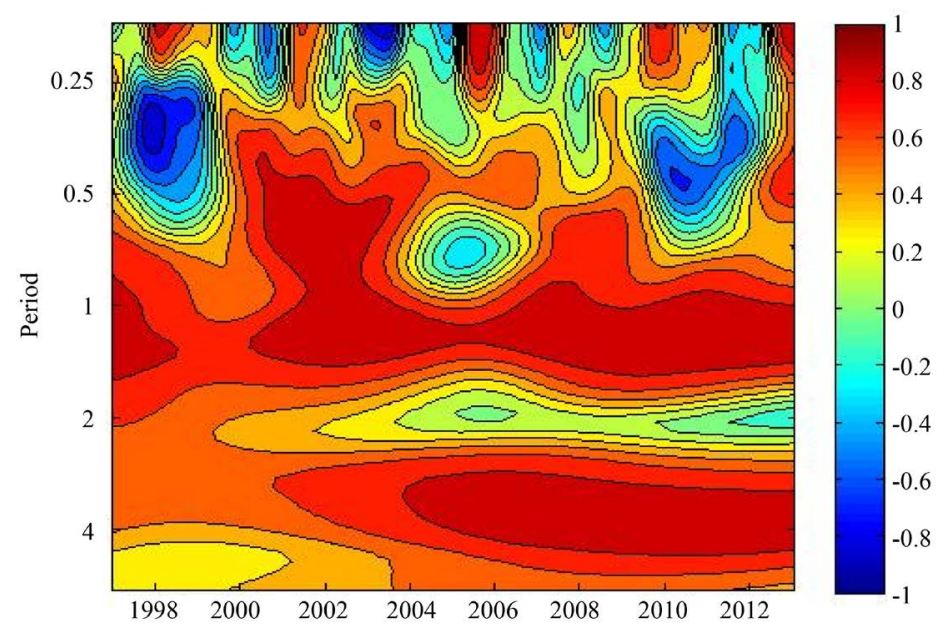

Figure 6. Co-movement between the uncertainty indices of Germany and UK.

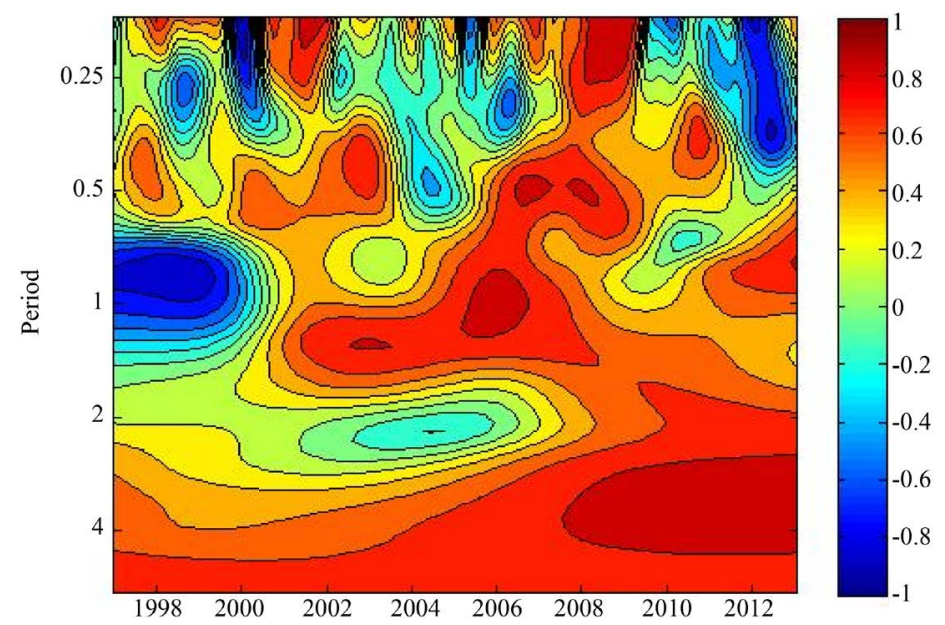

Figure 7. Co-movement between the uncertainty indices of Italy and UK. 
The results based on his analysis show several interesting findings. It is observed that there are higher comovements at intermediate and lower frequencies for all the studied pair of countries (France and Germany being exceptional to it). The co-movement of uncertainty is very high at business cycle frequencies (2 - 8 years) for all the country pairs. A note worthy finding is that the uncertainty indices are highly synchronized during the subprime mortgage crisis of 2007-08 for all the country pairs. This synchronization extends from mid 2007 (the time of origin of subprime crisis) till 2012 at lowest frequencies for all the country pairs. For some country pairs like France-Germany, France-Italy France-UK and Italy-UK, the synchronization of uncertainty extends to lower higher frequencies also. Another interesting point is that co-movement is also very high during 2001-02 for almost all the country pairs. This co-movement ranges from higher frequencies to intermediate frequencies and could be probably because of the dotcom bubble burst in 2001-02. These findings indicate that uncertainty could be synchronized at different frequencies. Moreover, it unveils the fact that during the periods of crises there are more chances of uncertainty spillovers.

\section{Conclusion}

We have analyzed the uncertainty spillovers over time and across frequencies in selected European countries. Using the new uncertainty index from Baker et al. [1] we estimated the wavelet based measure of cohesion to unravel both the time and frequency dependence in the co-movement of uncertainty. Broadly the study observed the following facts. Firstly, the co-movement of uncertainty found to be both time dependent as well as frequency dependent. In other words, uncertainty is observed to vary across frequencies and over time. Second, it was observed that the uncertainty indices mostly co-move at intermediate and lower frequencies for almost all the pairs of countries implying uncertainty are synchronized over medium to long run. Third, during the crises period of 2000-01 and 2007-08 the co-movement of uncertainty observed to be more pronounced and to vary at different frequencies.

\section{References}

[1] Baker, S., Bloom, N. and Davis, S. (2013) Measuring Economic Policy Uncertainty. Working Paper, Stanford University, Stanford.

[2] Rua, A. (2010) Measuring Co-Movement in the Time-Frequency Space. Journal of Macroeconomics, 32, 685-691. http://dx.doi.org/10.1016/j.jmacro.2009.12.005

[3] Neely, C.J. and Rapach, D.E. (2008) International Co-Movements in Inflation Rates and Country Characteristics. Working Paper 2008-025F, Federal Reserve Bank of St. Louis Research Division, St. Louis.

[4] Kiviaho, J., Nikkinen, J., Piljak, V. and Rothovius, T. (2012) The Co-Movement Dynamics of European Frontier Stock Markets. European Financial Management, 20, 574-595. http://dx.doi.org/10.1111/j.1468-036X.2012.00646.X

[5] Rua, A. and Nunes, L. (2009) International Co-Movement of Stock Market Returns: A Wavelet Analysis. Journal of Empirical Finance, 16, 632-639. http://dx.doi.org/10.1016/j.jempfin.2009.02.002

[6] Madaleno, M. and Pinho, C. (2012) International Stock Market Indices Co-Movements: A New Look. International Journal of Finance and Economics, 17, 89-102. http://dx.doi.org/10.1002/ijfe.448

[7] Percival, D.B. and Walden, A.T. (2000) Wavelet Methods for Time Series Analysis. Cambridge Press, Cambridge. http://dx.doi.org/10.1017/CBO9780511841040

[8] Croux, C., Forni, M. and Reichlin, L. (2001) A Measure of Co-Movement for Economic Variables: Theory and Empirics. Review of Economics and Statistics, 83, 232-241. http://dx.doi.org/10.1162/00346530151143770 